\title{
Hybrid superprism with low insertion losses and suppressed cross-talk
}

\author{
Jeremy Witzens, Thomas Baehr-Jones, and Axel Scherer \\ Department of Electrical Engineering, California Institute of Technology, Mail Code 136-93, Pasadena, California 91125, USA \\ (Received 15 July 2004; revised manuscript received 13 October 2004; published 11 February 2005; corrected 10 March 2005)
}

\begin{abstract}
We demonstrate with the two-dimensional finite-difference time-domain method that an adiabatic transition in a superprism with an interface along the $\left[\begin{array}{ll}1 & \overline{2}\end{array}\right]$ direction enhances the transmission through the superprism to more than $90 \%(-0.5 \mathrm{~dB})$ over the wavelength range $1.47-1.68 \mu \mathrm{m}$, including the telecommunication $C$ and $L$ bands. We also show that diffraction governed by a quasinegative index of refraction inside the superprism can be used to obtain nearly transform-limited beam widths at the output of the superprism. The reduction of the beam width at the output suppresses cross-talk and greatly enhances the achievable frequency resolution of the superprism.
\end{abstract}

DOI: 10.1103/PhysRevE.71.026604

PACS number(s): 42.82.Gw

\section{INTRODUCTION}

Photonic crystals (PC) [1] have attracted a lot of attention due to their ability to control the flow of light on a very small length scale and to tailor the dispersive properties of thin dielectric slabs. One class of PCs, planar photonic crystals [2] (PPC), represents particularly promising structures for integrated optics due to the fact that their planar fabrication allows for the use of conventional microelectronics patterning techniques. A PPC is an optically thin dielectric film perforated with a two-dimensional (2D) lattice of holes. Light is confined in the slab in the vertical direction by means of total internal reflection. The 2D lattice of holes can be used to confine light in the lateral direction by distributed Bragg reflection for applications such as ultrasmall high- $Q$ cavities $[3,4]$, ultrasmall lasers $[5,6]$, or photonic crystal waveguides $[7,8]$. For these applications, the useful frequency range of light lies within the photonic band gap. On the other hand, outside of the photonic band gap, the dispersive properties of the PC yield effects such as selfcollimation [9-14] and the superprism effect [15].

The superprism effect has been predicted to enable small planar wavelength demultiplexers [16-19]. However, in order to achieve practical devices, limitations such as insertion losses have to be overcome [20,21]. In this paper, we investigate a type of superprism with an enhanced frequency resolution, but in which insertion losses are also very high in the absence of a mode-matching mechanism, due to the fact that the structure of the PC Bloch-mode structure undergoes an abrupt change in the middle of the useful frequency range. However, we are able to achieve a high insertion efficiency on the order of $95 \%$ throughout a wide frequency range by adiabatically reducing the hole size at the PC interfaces. The two interfaces of the PC are along the $\left[\begin{array}{ll}1 & \overline{2}\end{array}\right]$ crystallographic axis [Fig. 1(a)], and the hole size is adiabatically reduced along the direction orthogonal to the interface (the [2 1$]$ direction). Inside the adiabatic transition region, holes that are offset by the $\left[\begin{array}{ll}1 & \overline{2}\end{array}\right]$ vector have the same size. Due to its adiabatic nature, this design does not need to be carefully fine-tuned as in $[20,21]$ and can be easily adapted to different lattices. In Sec. II, we will show that the adiabatic transition projects the complex mode structure of the PC Bloch modes onto a single plane wave.
In Sec. III, the issue of superprism compactness versus frequency resolution is addressed. In the previous literature, in order to evaluate the frequency resolution of the superprism, it has been assumed that a transform-limited beam is launched into the superprism [19] by terminating a waveguide right at the boundary of the PC. In a $k$-space power spectrum the phase information is lost, so that the spatial width of a beam can only be deduced from it if assumptions are made on the phases. A beam is transform-limited when its spatial width is the minimum possible width for its $k$-space power spectrum, and a Gaussian beam is transformlimited at its waist. Here we use the negative index of refraction $[22,23]$ in PCs [24-26] to enhance the frequency resolution capabilities of the superprism. Instead of injecting light into the PC by terminating a waveguide at its boundary, we terminate the waveguide some distance before and let the resulting beam expand in the unpatterned slab before it is transferred into the PC. The positive refraction in the unpatterned slab and the negative refraction in the PC can cancel each other out to a large extent so as to obtain transformlimited beams at the output facet of the PC. The criteria for frequency demultiplexion is that beams corresponding to different frequencies are spatially separated at the output boundary of the PC. When the beam widths at the output edge of the PC are smaller, less beam-steering and thus a smaller frequency change are necessary to separate the beams.

Furthermore, in this paper the waveguide leading to the PC has a steep angle relative to the boundary of the PC, so that light is coupled to PC Bloch modes through Fourier components in higher-order Brillouin zones (BZs). This has consequences that will be outlined in this section.

In the following, we investigate a 2D square lattice PC with the 2D finite-difference time-domain method [27] (FDTD). The refractive index of the slab is assumed to be 3.43 (silicon) and the holes are assumed to be backfilled with silicon dioxide (refractive index 1.46). The lattice-constant of the crystal $(a)$ is $0.4 \mu \mathrm{m}$ and the radius of the holes in the bulk part of the crystal (that is outside the adiabatic transition) is $0.12 \mu \mathrm{m}$. All fields are transverse-electric (TE) with the $B$-field pointing out of plane. The discretization is 0.01 $\mu \mathrm{m}$ and the time step $0.005 \mu \mathrm{m}^{-1}$ (in units of $c_{0}=1$, where $c_{0}$ is the speed of light in vacuum). 


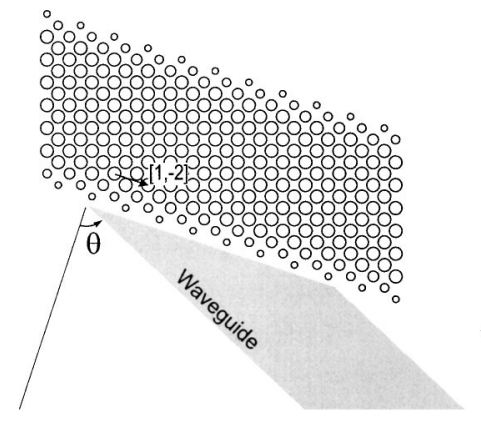

(a)

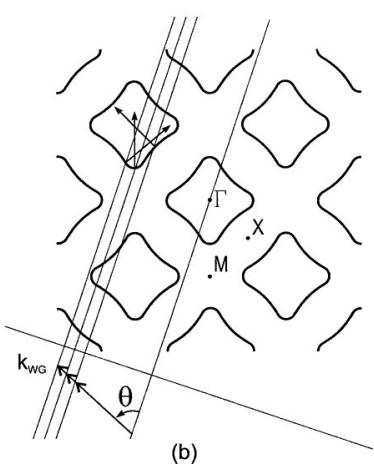

(b)

FIG. 1. In (a) the superprism configuration is illustrated. The waveguide direction relative to the PC as well as the orientation of the PC interfaces are shown. The hole size is varied in the adiabatic transition regions. Holes offset by the [ $1 \overline{2}]$ vector have the same radius. (b) illustrates the coupling condition from the waveguide to the photonic crystal. The $k$ vector of the waveguide mode $k_{\mathrm{WG}}$ is projected onto the equifrequency contour of the PC, with a projection direction perpendicular to the interface. At wavelengths shorter (higher) than $\lambda$ $=1.54 \mu \mathrm{m} k_{\mathrm{WG}}$ increases (decreases), and light is coupled to modes left (right) of the cusp. The direction of propagation is indicated by arrows inside the equifrequency contour. The $\Gamma, M$, and $X$ high-symmetry points are also shown.

Figure 2(a) shows the band diagram for this crystal along the $\Gamma M$ direction and Fig. 2(b) shows the equifrequency contour of the second photonic band at a free-space wavelength ( $\lambda$ ) of $1.54 \mu \mathrm{m}$. The equifrequency contour is the set of $k$ vectors corresponding to a given wavelength, and is the equivalent of the Fermi surface in solid-state physics. It can be seen that the equifrequency contour is approximately a square with rounded corners (the "cusps" of the contour). In the corner region, the group velocity $\vec{\nabla}_{\vec{k}} \omega$, perpendicular to the contour, undergoes a strong shift in direction. The rounded corner can be approximated as an arc of a circle as shown in Fig. 2(b). The radius of that circle is approximately $0.3 \times 2 \pi / 1.54$ so that the diffraction of a Gaussian beam inside the PC at that wavelength can be predicted by an effective index of -0.3 , provided the range of $k$ vectors of the beam is restricted to the corner region approximated by the arc. The negative sign in the effective index comes from the fact that the contour corresponds to the second band, folded back once into the first Brillouin zone (BZ), so that the group velocity points inside the contour. Negative refraction in the PC and positive refraction in the unpatterned slab can compensate each other, so that a beam diffracting in the slab can be refocused inside the PC.

In a superprism, an angled waveguide is coupled to a PC. In order to predict to which Bloch mode the waveguide is going to couple, the conservation of the $k$-vector component parallel to the interface $\left(k_{\|}\right)$is used [Fig. 1(b)]. Due to the discrete translation symmetry along the PC interface, this conservation is only exact modulo $2 \pi / A$, where $A$ is the periodicity of the $\mathrm{PC}$ along the interface. The superprism we investigate in this paper has both its interfaces along the $\left[\begin{array}{ll}1 & \overline{2}\end{array}\right]$ direction so that in this case $A=\sqrt{5} a . k_{\|}$can be deduced from the waveguide geometry by $k_{\|}=\sin (\theta) 2 \pi n_{\text {eff }} / \lambda$, where $n_{\text {eff }}$ is the effective index of the waveguide and $\theta$ is the angle between the waveguide and the normal to the interface of the PC [Fig. 1(a)]. In this paper, $\theta$ is chosen in such a way as to couple light at $\lambda=1.54 \mu \mathrm{m}$ to a Bloch mode located at the center of the cusp. This corresponds to the condition $k_{\|}$

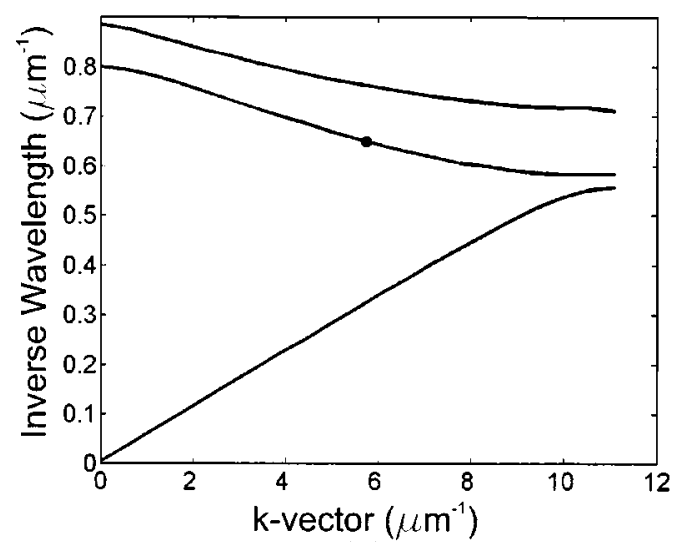

(a)

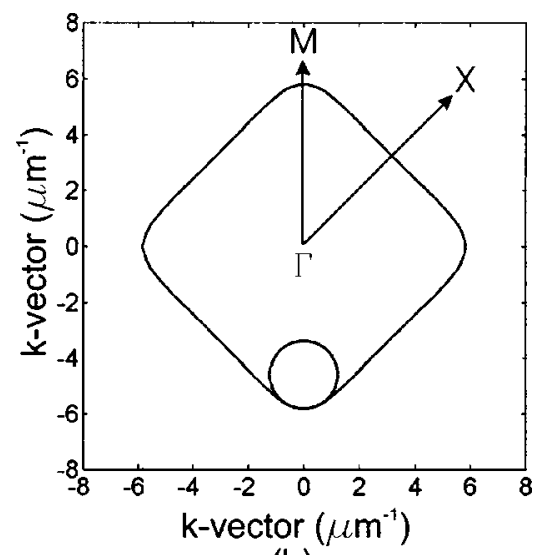

(b)

FIG. 2. (a) Band diagram showing the first three TE photonic bands along the $Г M$ direction. The black dot corresponds to $\lambda$ $=1.54 \mu \mathrm{m}$. The lattice constant of the PC was chosen in such a way that only the second band is present in the frequency range of interest ( $C$ band). (b) shows the equifrequency contour of the second band for $\lambda=1.54 \mu \mathrm{m}$. The contour has a squarish shape with rounded corners. The corners can be approximated by the arc of a circle with a radius of $0.3 \times 2 \pi / 1.54$, thus Gaussian beams with a distribution of $k_{\|}$ restricted to that area diffract inside the PC as if they were in a material of refractive index -0.3 . 


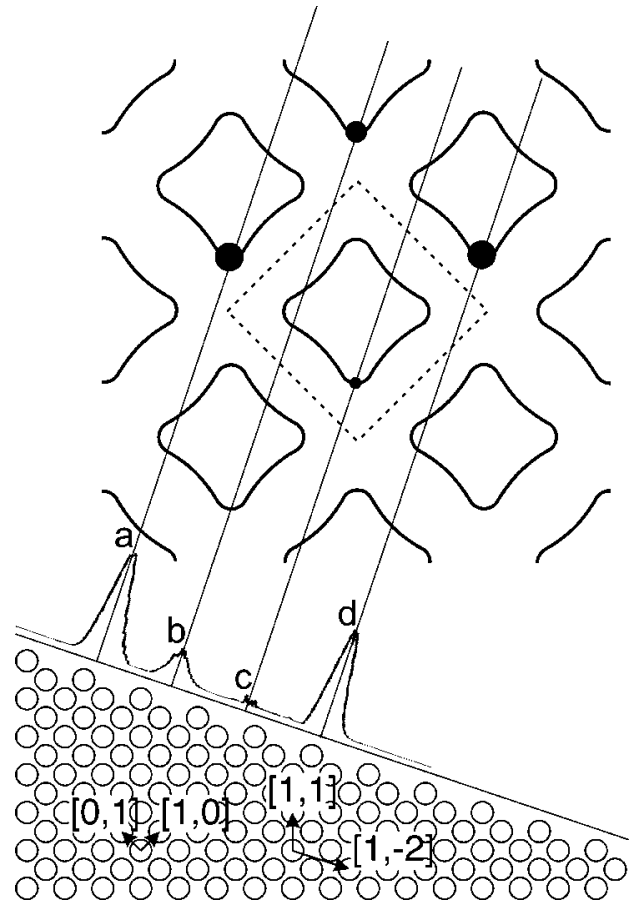

FIG. 3. This schematic illustrates how the different components of the Bloch mode project onto the interface between the crystal and the slab. The squarish contours show the equifrequency contour inside the first BZ (shown by the dashed square) and in higher Brillouin zones (outside the dashed square). The four main Fourier components of the Bloch mode located on the lower cusp as well as their projection on the interface of the crystal are shown by dots and by a curve along the interface. The curve corresponds to real data and gives an idea of the relative intensity of those components. They are spaced by $2 \pi /(a \sqrt{5})$ and labeled for reference in the text. Components $\{a, b, c, d\}$ correspond to $m=\{-2,-1,0,1\}$. It is apparent that component $a$, which we are coupling to as we chose $m=$ -2 , is one of the two dominant components. We also show the

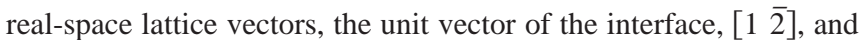
the direction of propagation of light at $\lambda=1.54$, [1 1 .

$=k_{\|, \text {cusp }}+m 2 \pi / A$, where $k_{\|, \text {cusp }}$ is the projection of the reduced $k$ vector (inside the first BZ) of the center of the cusp onto the interface, and $m$ is an integer. The projection of the various Fourier components of the Bloch mode onto the interface is shown in Fig. 3. $k_{\| \text {,cusp }}$ corresponds to the position of component $c$ in Fig. 3. Depending on the value of $m$, the light exciting the waveguide couples to the Bloch mode through a different Fourier component; $m=\{-2,-1,0,1\}$ correspond to Fourier components $a, b, c$, and $d$, respectively (the letters label the Fourier components, while the integer $m$ refers to a coupling condition, thus we keep the two separate notations). Here $m$ is chosen to be -2 so that we couple to a higher-order Fourier component (component $a$ in Fig. 3) outside the first BZ. This results in $\theta=60.95^{\circ}$.

The fact that we use $m=-2$, or in other words that we couple to a higher Fourier component outside the first BZ, has consequences in terms of beam steering and in terms of insertion efficiency. The mechanism leading to demultiplexion is slightly different from $m=0$ in that the beam-steering is not as much a consequence of a frequency-dependent de- formation of the equifrequency contour as of the fact that for different wavelengths a different part of the equifrequency contour is sampled. Of course both effects are present, but due to the steep injection angle the latter is dominant. At a lower (higher) wavelength, the value of $k_{\|}$increases (decreases) so that light is coupled left (right) of the cusp [Fig. 1(b)]. By moving from the left of the cusp to the right of the cusp, the group velocity undergoes a near to $90^{\circ}$ change in propagation direction. The large injection angle $\theta=60.95^{\circ}$ accelerates the change from one side of the cusp to the other side of the cusp, so that the frequency resolution of the superprism is higher for $m=-2$ than, for example, for $m=-1$ (component $b$ ), which corresponds to a smaller injection angle. The second reason why we preferred to work with $m=-2$ is that even in the absence of a mode-matching mechanism, much higher insertion efficiency is achievable this way due to the fact that we couple to a dominant component of the Bloch mode. As we will show in Sec. II, in the absence of a mode-matching mechanism the insertion efficiency $I_{x}$ corresponding to coupling through component $x$ $\in\{a, b, c, d\}$ can be approximated to a high degree of accuracy by

$$
I_{x}=P_{x} /\left(P_{a}+P_{b}+P_{c}+P_{d}\right),
$$

where $P_{x}$ is the fraction of the total power of the Bloch mode contained in component $x$ (this formula corresponds to a simple mode-overlap integral). It is then apparent that the insertion efficiency is proportional to the fraction of the Bloch mode contained in the Fourier component that is coupled to by the waveguide. As already mentioned, we are coupling to component $a$, which is one of the two dominant Fourier components of the Bloch mode. It would be very unfavorable to couple to component $c$ as it contains only a tiny fraction of the total power.

\section{ADIABATIC MODE CONVERSION}

In order to predict the propagation direction of a Bloch mode, it is sufficient to consider the shape of the equifrequency contour in the first BZ. However, in order to predict insertion losses at the PC boundary, the complex structure of Bloch modes with Fourier components in higher-order BZs has to be taken into account. The equifrequency contour in the first BZ arises from folding back the dispersion characteristic of the slab into the first BZ. However, most of the power of the Bloch modes is still contained in Fourier components close to the dispersion of the unpatterned slab, as schematically shown in Fig. 4. In particular, when moving along the equifrequency contour from one side of the squarish contour to another side of the contour, through the cusp, the mode structure undergoes a sharp change. On the left side of the corner the [ $\left.\begin{array}{ll}1 & 0\end{array}\right]$ component is dominant (component $d$ in Fig. 3), while on the other side the [l $\left.\begin{array}{ll}0 & 1\end{array}\right]$ component (component $a$ ) is dominant [21]. The mode that is exactly on the $\Gamma M$ direction has an equal fraction of its power in the $[1$ $0]$ and in the [l $\left.\begin{array}{ll}0 & 1\end{array}\right]$ components. Both components have to be provided at the interface to the PC to achieve good coupling to the Bloch mode. For example, in the case $m=-2$ investigated in this paper, in the absence of mode matching the 


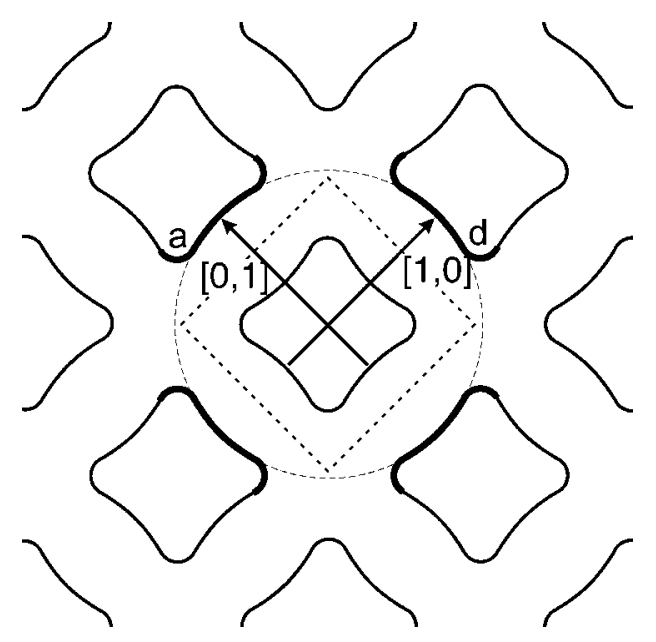

FIG. 4. This schematic illustrates the Fourier structure of the Bloch modes. The equifrequency contour originates from folding back the equifrequency contour of the slab into the first BZ. The first BZ is shown by the dashed square and the dashed circle represents the dispersion characteristic of the unpatterned slab. However, in extended Fourier space, most of the power of the mode is still contained in the higher-order components that are close to the original contour. These components are indicated by a thickened line along the higher-order contours and are labeled by $a$ and $d$ as in Fig. 3. They are offset from the component in the first BZ by inverse lattice vectors represented by arrows and labeled by $\left[\begin{array}{ll}0 & 1\end{array}\right]$ and $\left[\begin{array}{ll}1 & 0\end{array}\right]$. Of course this model is a simplification, as the lattice of holes not only perturbs the contour at the anticrossing points, but also perturbs slightly the rest of the contour.

insertion efficiency is good for modes to the right of the cusp, however the insertion efficiency is worse for modes to the left of the cusp that are dominated by component $d$ rather than component $a$. The mode structure will be quantified below as well as a quantitative model given for the insertion losses.

In this section, we introduce an adiabatic transition that alleviates the mode-matching problem. Due to the fact that the $k$ vector of the Bloch modes that are coupled to varies with the frequency, the mode matching interface needs to be efficient not only for a wide frequency range, but also for a wide $k$-vector range. This is a difficult problem due to the fact that the Bloch-mode structure is $k$-vector-dependent; in particular, it is strongly dependent on which side of the cusp the Bloch mode is on. This problem is solved by the adiabatic transition for modes in the vicinity of the cusp. The principle of the adiabatic transition is illustrated in Fig. 5(a). The hole size is progressively decreased inside the adiabatic transition region. The main effect of decreasing the hole size is a shrinking of the equifrequency contour. This can be explained to the first order by an increase of the effective index of the PC slab due to a higher volume ratio of high index material, so that the unfolded contour (dashed circle in Fig. 4) is enlarged. After folding back the contour into the first $\mathrm{BZ}$, the resulting squarish contour is smaller. In other words, due to the fact that the second band is folded back once, the dependence of the size of the contour on the index of the materials constituting the slab is the opposite of what would be expected from an unpatterned slab. Due to the slanted

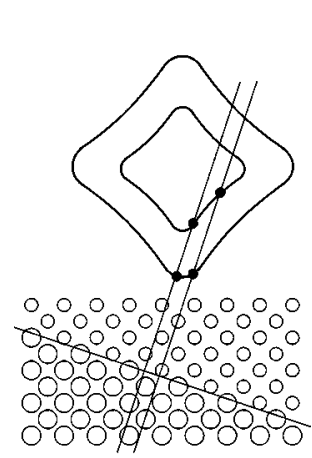

(a)

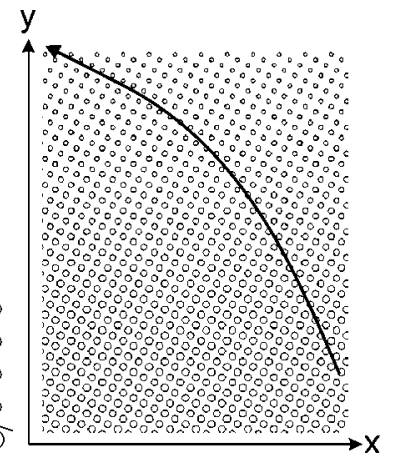

(b)
FIG. 5. (a) illustrates the mechanism underlying the adiabatic transition. To simplify the schematic, we show a step in hole radius rather than a continuous change, but the general idea is the same. Due to the change in hole size, the contour is deformed. In particular, for the same wavelength, the contour in the region of smaller holes is smaller. Because the translation invariance is conserved along the $\left[\begin{array}{ll}1 & \overline{2}\end{array}\right]$ direction, it is straightforward to predict how a Bloch mode couples from one crystal to the other by projecting it along the [2 1] direction. Modes that were in the vicinity of the cusp in the "bulk" PC (with larger holes) couple to modes on the side of the contour in the PC with smaller holes. Those modes have a dominant Fourier component and are easy to couple to. When the transition is made adiabatic, rather than stepwise, the reflections induced at the interface are suppressed. (b) illustrates the real-space behavior of the light propagation. In the adiabatic transition region, the group velocity is progressively deflected. The interface of the PC is along the $x$ direction [the coordinate frame of (b) is rotated relative to the other figures].

orientation of the interface, the Bloch modes originally in the vicinity of the cusp are not projected onto the cusp of the smaller contour (as would happen if the interface had been along the $\left[\begin{array}{ll}1 & \overline{1}\end{array}\right]$ direction and the hole size varied along the $[1$ 1] direction). Rather, while propagating through the adiabatic region, the Bloch modes originally in the vicinity of the cusp are progressively projected onto the side of the smaller squarish contours corresponding to the local band structure. On the sides of the contour, the Bloch modes are dominated by a single Fourier component and are easy to couple to. Furthermore, with decreasing hole size this single Fourier component dominates more and more the overall Fourier structure until $100 \%$ of the mode is contained in this single component. In order to simplify the diagram, Fig. 5(a) shows a step change of the hole radius instead of a continuous change over a large number of layers. Such a brusk change would induce reflections as well as out-of-plane scattering losses in a planar PC defined in a finite thickness slab. The slow transition from the full hole size to the small holes in Fig. 5(b) suppresses these loss mechanisms. It is essential for the interface to be along an orientation other than $\left[\begin{array}{ll}1 & \overline{1}\end{array}\right]$ when modes on both sides of the cusp are being mode-matched. Indeed, if the orientation were $\left[\begin{array}{ll}1 & \overline{1}\end{array}\right]$, the mode on the center of the cusp would stay at the center of the cusp throughout the mode-matching interface, and would finally be converted to two plane waves. 


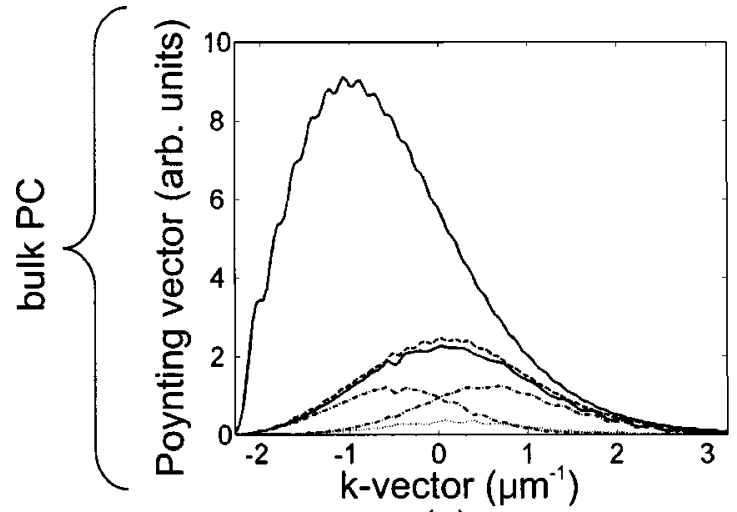

(a)

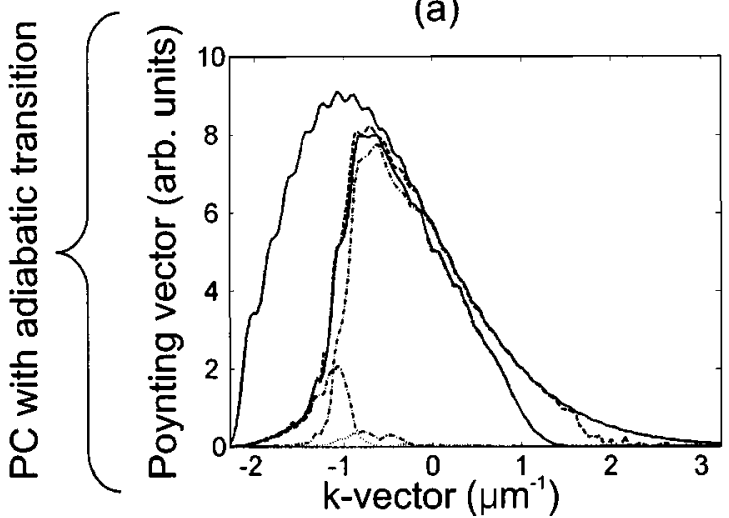

(c)

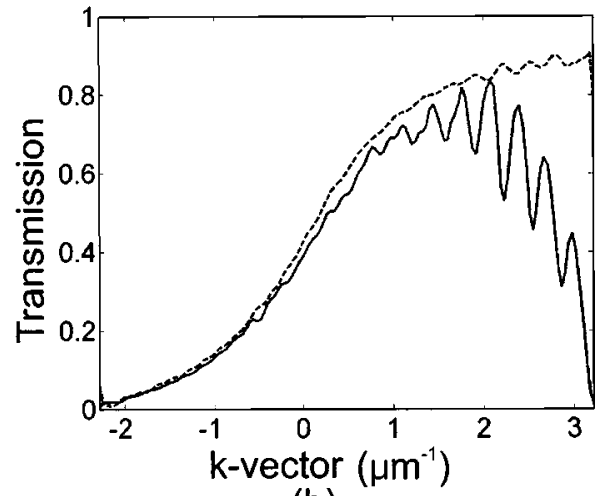

(b)

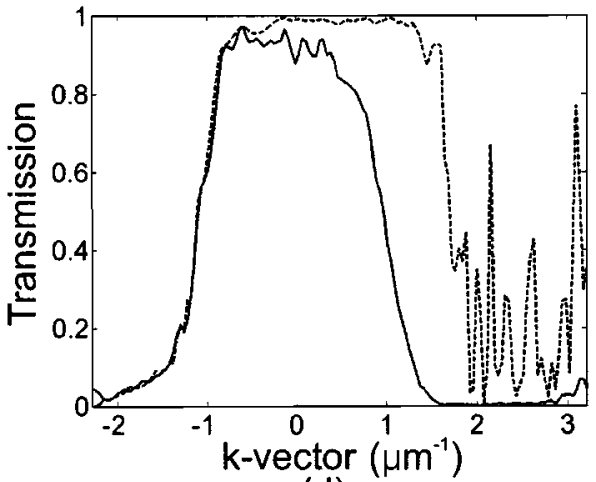

(d)

FIG. 6. We propagate light at $\lambda=1.54 \mu \mathrm{m}$ through a bulk PC [(a) and (b)] and through an adiabatic PC [(c) and (d)]. The abscissae correspond to the projection onto the interface of the reduced $k$ vector of the Bloch mode (the zero abscissa has been shifted so that it corresponds to the mode on the $\Gamma M$ direction). In (a) and (c), the upper continuous curve is the incoming power and the lower continuous curve the outgoing power. The dashed curve is the prediction of the output power based on Eq. (1). The left dash-point curve corresponds to component $d$ (Fig. 3), the right dash-point curve to component $a$, and the pointed curve to component $b$. All the Fourier components corresponding to the same Bloch mode are represented at the same abscissa. In (b) and (d), we show both the actual insertion efficiency (continuous curve) and the predicted insertion efficiency based on Eq. (1) (dashed curve). In (a) and (c), the trailing edge of the incoming Gaussian beam is due to the fact that the interface of the PC is rotated with respect to the direction of propagation of the beam.

Figure 5(b) illustrates the real-space behavior of light in the slab, superprism, and adiabatic transition region. In the latter, the direction of propagation undergoes a change progressively. This is an additional advantage over the modematching interface introduced in Ref. [21]. Indeed in [21] the two Fourier components containing most of the power of the Bloch mode were progressively generated, but had different directions of propagation inside the mode-matching interface, so that the interface had to be compact to avoid the two components spatially separating. In the adiabatic transition region, the light corresponds to the local Bloch mode at each point of the transition, so that all the generated components copropagate in the same direction. In particular, in [21] there was a tradeoff between compactness and insertion efficiency which is not an issue in the present design, as there is no disadvantage in increasing the size of the adiabatic transition other than a slight loss of real estate on the chip.

We evaluated the insertion efficiency of the adiabatic transition by 2D FDTD computation. In the following simulations, we adiabatically reduced the hole radius from $0.12 \mu \mathrm{m}$ to $0.06 \mu \mathrm{m}$. We did not go all the way to zero for practical reasons, both due to limitations of the FDTD method (discretization) and due to the resolution limitations of lithogra- phy in a fabricated device. We transmit light through PCs with both interfaces along the $[2 \overline{1}]$ direction. In one case the PC consists in a "bulk" PC and in the other case by a PC with adiabatic transitions at the input and at the output facets (the "adiabatic PC"). The bulk PC is 150 layers wide, where "layer" refers to a row of holes along the [2 1 ] direction [for example, the PC in Fig. 1(a) would correspond to 23 rows]. The adiabatic PC consists of 150 layers, where the hole radius is ramped up from $r=0.06 \mu \mathrm{m}$ to $r=0.12 \mu \mathrm{m}$ in the first 50 layers, kept constant in the next 50 layers, and finally ramped back down to $r=0.06 \mu \mathrm{m}$ in the last 50 layers. In Fig. 6, we show data for a wide range of $k$ vectors but for a fixed wavelength $\lambda=1.54 \mu \mathrm{m}$. A transform-limited Gaussian beam is launched in the unpatterned slab before the PC, with an angle $\theta=60.95^{\circ}$ relative to the normal to the interface of the PC, as derived in Sec. I. However, the waist of the beam is chosen to be narrow; due to the small waist, the light source contains a wide range of $k$ vectors centered on $k_{\|, \text {cusp }}+m 2 \pi / A$ (with $m=-2$ ), rather than a narrow $k$-vector distribution generated by a wider light source, so that data can be collected for a wide range of $k$ vectors. The field at the output facet of the PC is Fourier-transformed, and the component of the Poynting vector along the $y$ direction 
$P_{y}\left(k_{\|}\right)$is calculated as a function of $k_{\|}$. Three items are confirmed by these data: First, the Bloch-mode structure indeed undergoes the predicted transition from one Fourier component to the other; second, Eq. (1) is adequate to predict the insertion losses in the vicinity of the cusp and in the absence of an adiabatic transition; and third, the adiabatic transition does solve the insertion problem for a wide range of $k$ vectors. In order to evaluate the insertion efficiency, in both cases we compare the incoming power to the transmitted power. In order to show the Bloch-mode structure, we decompose the transmitted power into the various Fourier components. Finally, to verify the validity of Eq. (1), we compare the actual transmitted power to the transmitted power predicted by Eq. (1).

Figures 6(a) and 6(c) show the raw data, respectively, for the bulk PC and for the adiabatic PC. Figures 6(b) and 6(d) show the actual transmission through the PC as well as the predicted transmission. In Fig. 3, we can see that there are four peaks in the $k$-space transmission spectrum, three of which, $a, b$, and $d$, are significant. In Figs. 6(a) and 6(c), we show $a$ (rightmost dash-pointed curve), $b$ (pointed curve), and $d$ (leftmost dash-pointed curve) as a function of their reduced $k$ vector, so that all the components corresponding to the same Bloch mode share the same abscissa. In other words, the Fourier components corresponding to $k_{\|}+p 2 \pi / A$ with $p \in\{-2,-1,0,1\}$ are all plotted with the abscissa $k_{\|}$. In all four figures, the zero abscissa corresponds to the intersection of the contour with the $\Gamma M$ direction, the center of the cusp. The total outgoing power is the sum of those three components and is shown by the lower continuous curve. The incoming power is shown by the upper continuous curve. The dashed curve, almost identical to the outgoing power, corresponds to the outgoing power predicted by Eq. (1) from the power distribution in components $a, b$, and $d$. In Figs. 6(b) and 6(d), the continuous curve is the actual transmission, that is, the outgoing power divided by the incoming power, while the dashed curve is the predicted transmission.

There are some comments that can be made on these data. In Fig. 6(a), the Bloch-mode structure predicted by the model shown in Fig. 4 can be clearly seen. Modes to the left of the cusp, with a negative abscissa, propagate to the right and the dominant component is $d$. Conversely, modes to the right of the cusp, with a positive abscissa, propagate to the left and the dominant component is $a$. In Fig. 6(b), it can be seen that the predicted insertion efficiency is S-shaped, where modes with a higher abscissa, that are dominated by component $a$, have a higher insertion efficiency because component $a$ is provided by the light source in the slab. The actual insertion efficiency is close to the predicted one for a large range of $k$ vectors. At positive abscissae there is also another limiting mechanism on the insertion efficiency that we did not investigate. In the case of the adiabatic PC, as predicted, component $a$ is heavily dominant in the transmission spectrum around the zero abscissa. This leads to an insertion efficiency above $90 \%$ for a large range of $k$ vectors on both sides of the cusp (i.e., with positive and negative abscissae). Also, it can be seen that the transition point from one dominant Fourier component to the other is shifted from $k_{\|}=0$ to $k_{\|}=-1.12 \mu \mathrm{m}^{-1}$. That is to be expected from the model shown in Fig. 5(a), where a mode originally left of the

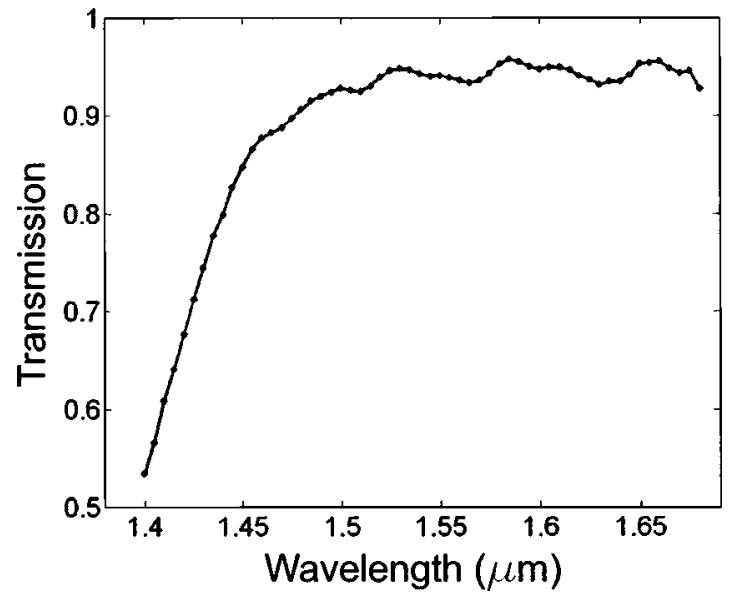

FIG. 7. Power transmission through the "adiabatic crystal." A Gaussian beam with a FWHM of $3 \mu \mathrm{m}$ and an angle $\theta=60.95^{\circ}$ is launched in the slab and coupled to the crystal (150 layers, 100 of which are split between the adiabatic transitions on both interfaces). $\theta$ is chosen so that light at $\lambda=1.54 \mu \mathrm{m}$ propagates along the [1 1 ] direction. The transmission is in excess of $90 \%$ over the wavelength range $1.47-1.68 \mu \mathrm{m}$.

cusp (with $k_{\|}=-1.12 \mu \mathrm{m}^{-1}$ ) gets projected onto the cusp of the smaller contour, while all of the modes to its right, including the mode originally on the cusp (with $k_{\|}=0$ ), get projected on the side of the contour where $a$ is dominant. Finally, in the case of the adiabatic PC, the transition from one Fourier component to the next is much more abrupt, as visualized by the slope at $k_{\|}=-1.12 \mu \mathrm{m}^{-1}$ in Fig. 6(d). This is due to the fact that the field at the output of the crystal corresponds to the modes of the PC with reduced hole size $(r=0.06 \mu \mathrm{m})$, where the coupling strength between Fourier components as well as the extent in $k$ space over which anticrossing occurs are reduced.

As explained in detail in Ref. [19], for an angled waveguide coupled to a PC, there is a one-to-one relationship between the wavelength and the $k$ vector of the Bloch mode that is coupled to. The true figure of merit of a modematching interface used in conjunction with a superprism is the insertion efficiency at each of these frequency or $k$-vector points, that is, the frequency-dependent coupling efficiency of the complete system. We show these data in Fig. 7. The field is launched from a much wider Gaussian beam, the full width at half minimum (FWHM) is $3 \mu \mathrm{m}$, so that a narrow range of $k$ vectors is targeted at each frequency, rather than averaging the insertion efficiency over a wide range of $k$ vectors. The Gaussian beam approximates the mode profile of the waveguide leading to the superprism in a real device. It can be seen that the insertion efficiency (transmittance through two interfaces with adiabatic transition) is above $90 \%$ for a range of wavelengths $(1.47-1.68 \mu \mathrm{m})$ including the telecommunication $C$ band $(1.528-1.570 \mu \mathrm{m})$ and $L$ band $(1.570-1.605 \mu \mathrm{m})$.

\section{COMPACTNESS AND SENSITIVITY}

In this section, we address the frequency sensitivity of the superprism. In Ref. [19] it was shown that the small radius of 


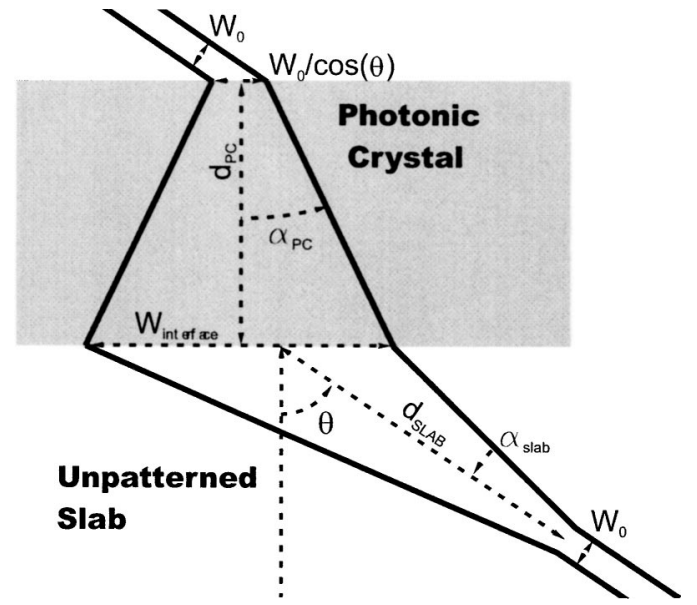

FIG. 8. This figure is a schematic of the beam expansion inside the unpatterned slab and inside the PC. The lengths and angles are labeled for reference in the text.

curvature at the corner of the equifrequency contour not only leads to beam-steering, but also to enhanced diffraction of the beam inside the PC. In other words, the beam coupled from the waveguide not only has a frequency-dependent direction of propagation inside the $\mathrm{PC}$, but also experiences stronger beam-broadening than in the unpatterned slab. In order to separate frequencies with low cross-talk, the corresponding beams should have vanishing spatial overlap at the output facet of the PC. Due to beam-broadening, the spatial separation necessary to separate the beams is higher, so that PC induced enhanced diffraction reduces the frequency resolution of the superprism. The overall efficiency in terms of frequency selectivity was quantified in [19], however the model developed assumed that the beam at the input interface of the PC was transform-limited, that is, the waist of the beam is assumed to be at the interface to the PC. This situation is encountered when the waveguide stops exactly at the interface to the PC. Here, however, we examine the situation where the waveguide stops some distance before the interface, so that the beam diffracts inside the unpatterned slab before coupling into the PC. The diffraction inside the PC resembles negative index diffraction, so that the diffraction inside the unpatterned slab and inside the PC can compensate each other. If the diffraction length in the unpatterned slab is adequately chosen, the beam can be nearly transform-limited at the output facet of the PC, so that the impact of beambroadening on the superprism resolution can be neglected (however, there is still a price to be paid to compensate for the enhanced diffraction inside the superprism, and that is the chip space necessary to expand the beam prior to the PC).

In the following, we derive the diffraction length in the slab $\left(d_{\text {slab }}\right)$ necessary to compensate the diffraction length inside the PC $\left(d_{\mathrm{PC}}\right)$. The width of the adiabatic transition has to be split in some way between $d_{\text {slab }}$ and $d_{\mathrm{PC}}$ that we do not quantify here, however in a real device the width of the adiabatic transition should be much smaller than both the width of the bulk part of the PC and the diffraction length inside the slab, so that it can be neglected. As shown in Fig. $8, d_{\mathrm{PC}}$ is the width of the PC (because we optimize $d_{\text {slab }}$ for the wavelength at which light propagates in the [2 1] direc- tion normal to the $\left[\begin{array}{ll}1 & \overline{2}\end{array}\right]$ interface) and $d_{\text {slab }}$ is the distance over which the beam is propagated inside the slab. We assume the beam to be a transform-limited Gaussian beam at the output edge of the waveguide and we aim for a transform-limited Gaussian beam at the exit of the superprism. The width of the beam at the end of the waveguide is $W_{0}$ [the width of the beam is defined as $2 \sigma$, where the functional dependence of the amplitude along the cross section of the beam is given by $\left.\exp \left(-x^{2} / \sigma^{2}\right)\right]$. The diffraction angle inside the unpatterned slab is $\alpha_{\text {slab }}=2 \lambda /\left(\pi n_{\text {slab }} W_{0}\right)$, so that the width of the beam at the input interface of the superprism is $W_{\text {interface }}=2 \tan \left(\alpha_{\text {slab }}\right) d_{\text {slab }} / \cos (\theta)$. The additional $1 / \cos (\theta)$ factor comes from the fact that $W_{\text {interface }}$ is the width along the interface of the PC that makes an angle $\theta$ with the normal to the propagation direction of the beam. The width of the transform-limited beam at the output facet of the PC should be $W_{0} / \cos (\theta)$, so that the diffraction angle inside the $\mathrm{PC}$ is $\alpha_{\mathrm{PC}}=2 \lambda \cos (\theta) /\left(\pi n_{\mathrm{PC}} W_{0}\right)$. Thus the size of the beam at the input facet of the PC can also be written as $W_{\text {interface }}$ $=2 \tan \left(\alpha_{\mathrm{PC}}\right) d_{\mathrm{PC}}$. By equating the two expressions for $W_{\text {interface, }}$ we get the equation

$$
\tan \left(\frac{2 \lambda \cos (\theta)}{\pi n_{\mathrm{PC}} W_{0}}\right) d_{\mathrm{PC}}=\tan \left(\frac{2 \lambda}{\pi n_{\text {slab }} W_{0}}\right) d_{\text {slab }} \frac{1}{\cos (\theta)} .
$$

Because the Gaussian beam expanding in the slab is cut at an angle $\theta$, the beam profile along the interface of the PC is not exactly Gaussian. Thus the model exposed above is an approximation. To further validate Eq. (2), we investigated the dependence of the width of the beam exiting the PC as a function of $d_{\text {slab }}$ with a simple beam propagation code based on a scalar description of the field, and an effective index approximation inside the PC. Here again the effective index approximation for the propagation inside the PC is only valid for a beam with a small enough $k$-vector distribution to be completely contained within the rounded part of the cusp. At the boundaries of the PC, an extra transformation has to be operated on the field that consists in subtracting $k_{\text {cusp,\| }}$ $+m 2 \pi / A$ from the lateral component of the $k$ vector $\left(k_{x}\right)$ at the input boundary, while at the output boundary it is added back to it. Indeed, inside the PC, light is described by an effective $k$ vector that corresponds to the offset between the center of the circle approximating the cusp of the equifrequency contour in the first $\mathrm{BZ}$ and the reduced $k$ vector of the Bloch mode. Light with a lateral $k$ vector of $k_{\text {cusp, } \|}$ $+m 2 \pi / A$ is in fact at the center of the cusp, and propagates inside the PC as if it had a 0 lateral $k$ vector in a material of index $n_{\mathrm{PC}}$. This model is a little more sophisticated than the simple considerations that led to Eq. (2), for example it takes into account that the field at the boundary to the PC is a distorted Gaussian due to the fact that the cross section of the incoming beam is taken along an oblique axis. For this example, the PC is assumed to be $300 \mu \mathrm{m}$ thick and the FWHM of the waveguide is assumed to be $5 \mu \mathrm{m}$. The optimum diffraction length in the slab is calculated to be $655 \mu \mathrm{m}$ with Eq. (2). In Fig. 9, we show the beam profile at the input boundary and at the output boundary of the PC, as a function

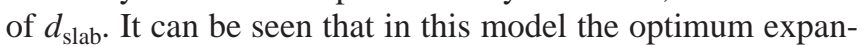
sion length is still given by Eq. (2). As mentioned in the first 


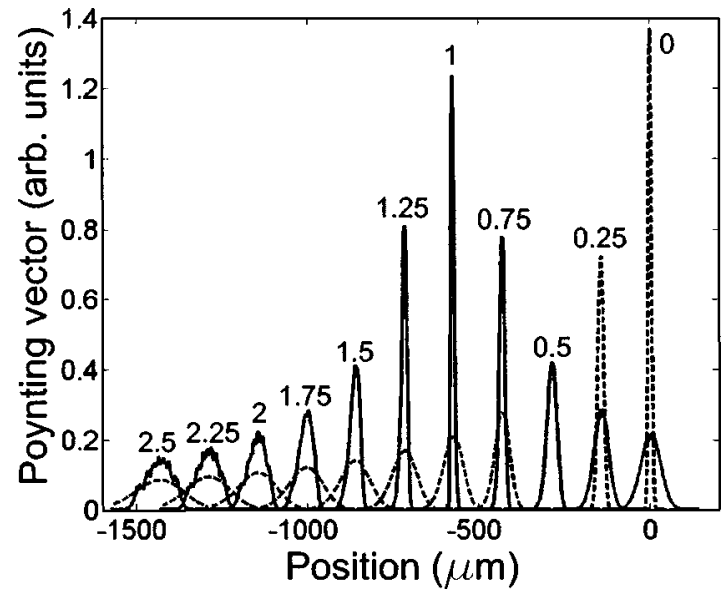

FIG. 9. This figure shows the dependence on the expansion length inside the unpatterned slab $\left(d_{\text {slab }}\right)$ of the beam width at the input (dashed line) and output (continuous line) boundaries of the PC. The beam profiles are labeled by $d_{\text {slab }} / d_{0}$, where $d_{0}$ is the optimum $d_{\text {slab }}$ predicted by Eq. (2). The frequency is assumed to be the one at which the beam propagates perpendicular to the PC interface inside the PC. At $d_{\text {slab }}=0$ (that is, the waveguide terminates directly in front of the PC interface), the beam is significantly broadened inside the PC. At the optimum $d_{\text {slab }}$, the diffractions inside the slab and inside the PC compensate each other and the beam has its minimum width at the PC output interface.

section, the diffraction of the beam inside the PC is described by an effective index $n_{\mathrm{PC}}=-0.3$ that is much smaller than the index of the silicon slab $\left(n_{\text {slab }}=3.43\right)$. As a consequence, the beam needs to propagate a longer distance in the slab than in the PC in order for the diffraction in the slab and in the PC to compensate each other. However, due to the fact that the beam propagates inside the slab along a slanted direction, the distance between the waveguide edge and the edge of the PC $[655 \cos (\theta)=318 \mu \mathrm{m}]$ is of the same order as the width of the PC $(300 \mu \mathrm{m})$.

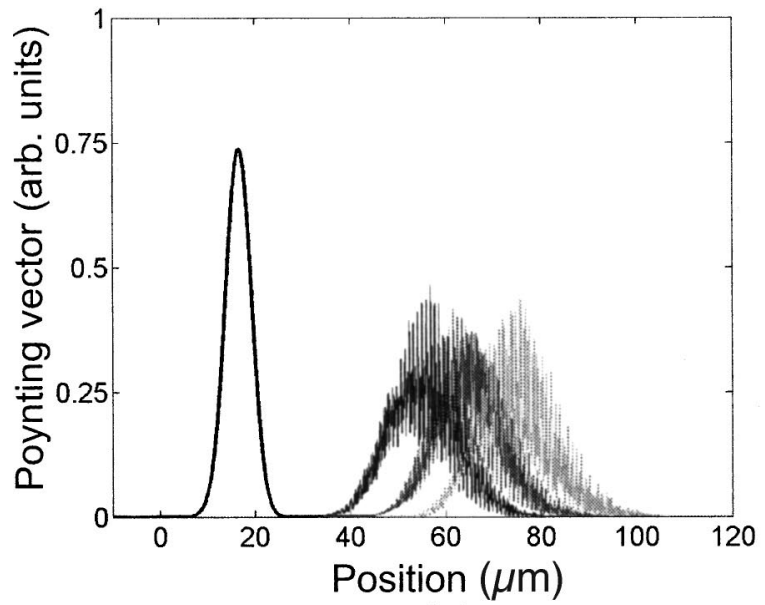

(a)
By applying Eq. (2), we also got convincing results with a full 2D FDTD simulation. We simulated the transmission through the adiabatic PC introduced in the previous section (same PC as in Figs. 6 and 7) by using as a light source a transform-limited beam at the input interface (a) and a beam that had been propagated $80 \mu \mathrm{m}$ before hitting the interface (b). The results are shown in Fig. 10. It can be seen that the separation between beams corresponding to different frequencies at the output of the superprism is much better in the second case. It can also be seen that the beam profile at the output interface in the second case is very close to the transform-limited beam profile.

We would like to finish with a few concluding remarks on the feasibility of a real device. The frequency resolution shown in Fig. 10 is $60 \mathrm{~nm}$ and might appear disappointing. However, this is uniquely due to the small size of the device simulated here; the PC is only $26.8 \mu \mathrm{m}$ wide. The beam width at the output of the PC is independent of the size of the $\mathrm{PC}$, as the beam is transform-limited due to the cancellation effect between positive refraction in the slab and negative refraction in the PC. However the separation between the center axis of beams corresponding to different frequencies is proportional to the size of the PC. Thus the resolution of the superprism scales with the size of the bulk part of the PC. For a $10 \mu \mathrm{m}$ separation (same as in Fig. 10) induced by a 1 $\mathrm{nm}$ wavelength difference, the necessary width can be evaluated to be $27 \times 60 / 1=1620 \mu \mathrm{m}$. This number is an overestimate as we scaled the bulk part of the PC and the adiabatic transition region by the same factor. In fact, beam steering is very weak in the small hole region, and we do not need to increase the width of the adiabatic region, so that the necessary PC size is probably more on the order of $1000 \mu \mathrm{m}$.

The calculations made in this paper were all 2D FDTD. As such they do not take into account that PC modes can be lossy in a planar photonic crystal (i.e., defined in a finite thickness slab) if they are inside the light cone. If the reduced $k$ vector of a Bloch mode has a smaller magnitude than

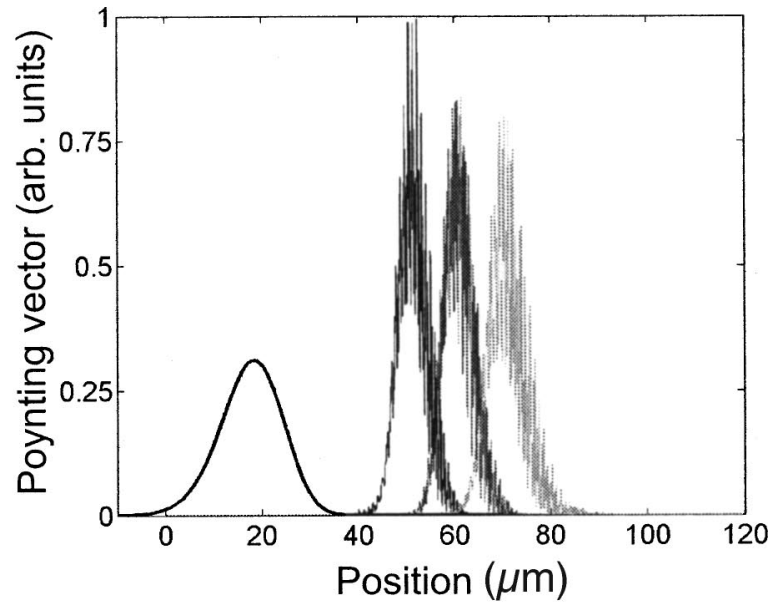

(b)

FIG. 10. In (a) a transform-limited beam (leftmost beam) is launched inside the PC. The shape of the beam at the output of the PC is shown for the free-space wavelengths $1.6 \mu \mathrm{m}, 1.54 \mu \mathrm{m}$, and $1.48 \mu \mathrm{m}$ (left to right). (b) shows the same data for a beam that has been propagated $80 \mu \mathrm{m}$ inside the slab before being coupled to the PC. The leftmost beam is the cross section of the beam at the input edge of the PC after propagation through the slab. In this case, the beam is approximately transform-limited at the output of the PC. Both in (a) and in (b), the relative positions of the output beams are correct, but the input beams have been separated out, so as not to overlay too many plots. 
$2 \pi n_{\text {cladding }} / \lambda$, where $n_{\text {cladding }}$ is the refractive index of the cladding material surrounding the planar photonic crystal (typically $\mathrm{SiO}_{2}$ for a $\mathrm{PC}$ made in a $\mathrm{Si}$ membrane), the Fourier component inside the first BZ can couple to propagating cladding modes, which leads to a loss mechanism. For extended devices such as the superprism where light has to propagate for a significant distance through the crystal, this leads to very large losses, if not to a complete signal loss. We investigated some band diagrams and found that a PC lattice with $a=480 \mathrm{~nm}$ and $r / a=0.3$ defined in a Si membrane clad with $\mathrm{SiO}_{2}$ is a good candidate. The $C$ band is just below the light line and the corresponding Bloch modes have no intrinsic losses. We chose to work as close as possible to the light line because the shape of the equifrequency contour is very close to the one investigated in this paper. The lattice constant of the PC is slightly bigger to compensate for the higher volume ratio of low index material $\left(\mathrm{SiO}_{2}\right.$ cladding the finite thickness film).

\section{CONCLUSION}

We have introduced two novel concepts to enhance the design of superprisms for wavelength demultiplexion. One is an adiabatic transition along the [2 1$]$ direction in a superprism with interfaces along the $\left[\begin{array}{ll}1 & \overline{2}\end{array}\right]$ direction. This solves the light insertion problem with a robust design, because the only necessary condition is a slow transition; variations in photonic crystal design and slab index should be easily accommodated. Also it yields insertion efficiencies in excess of $90 \%$ for a wide range of frequencies and $k$ vectors, making it uniquely suited to the superprism effect. The second improvement consists in using a quasinegative index of refraction to remove one of the limitations on frequency resolution. By ensuring that beams exiting the superprism are transform-limited, cross-talk is suppressed. Those two improvements may pave the way for the superprism to be used in practical wavelength demultiplexers.

\section{ACKNOWLEDGMENTS}

The authors wish to acknowledge generous support from DARPA under Contract No. N-00421-02-D3223, and the AFOSR under Contract No. F49620-03-1-0418.
[1] E. Yablonovitch, Phys. Rev. Lett. 58, 2059 (1987).

[2] T. F. Krauss, R. M. De La Rue, and S. Brand, Nature (London) 383, 699 (1996).

[3] K. Srinivasan, P. E. Barclay, and O. Painter, Opt. Express 12, 1458 (2004).

[4] Y. Akahane, T. Asano, B. S. Song, and S. Noda, Nature (London) 425, 944 (2003).

[5] O. Painter, R. K. Lee, A. Yariv, A. Scherer, J. D. O'Brian, P. D. Dapkus, and I. Kim, Science 284, 1819 (1999).

[6] T. Yoshie, M. Loncar, A. Scherer, and Y. Qiu, Appl. Phys. Lett. 84, 3543 (2004).

[7] Y. Désières et al., J. Appl. Phys. 92, 2227 (2002).

[8] M. Lončar et al., Appl. Phys. Lett. 80, 1689 (2002).

[9] H. Kosaka, T. Kawashima, A. Tomita, M. Notomi, T. Tamamura, T. Sato, and S. Kawakami, Appl. Phys. Lett. 74, 1212 (1999).

[10] D. N. Chigrin, S. Enoch, C. M. Sotomayor Torres, and G. Tayeb, Opt. Express 11, 1203 (2003).

[11] J. Witzens and A. Scherer, J. Opt. Soc. Am. A 20, 935 (2003).

[12] L. Wu, M. Mazilu, and T. F. Krauss, J. Lightwave Technol. 21, 561 (2003).

[13] C. Chen, A. Sharkawy, D. M. Pustai, S. Shi, and D. W. Prather, Opt. Express 11, 3153 (2003).

[14] X. Yu and S. Fan, Appl. Phys. Lett. 83, 3251 (2003).

[15] H. Kosaka, T. Kawashima, A. Tomita, M. Notomi, T. Tamamura, T. Sato, and S. Kawakami, Phys. Rev. B 58, R10096 (1998)
[16] H. Kosaka, T. Kawashima, A. Tomita, M. Notomi, T. Tamamura, T. Sato, and S. Kawakami, Appl. Phys. Lett. 74, 1370 (1999).

[17] T. Baba and M. Nakamura, IEEE J. Quantum Electron. 38, 909 (2002).

[18] L. J. Wu, M. Mazilu, T. Karle, and T. F. Krauss, IEEE J. Quantum Electron. 38, 915 (2002).

[19] T. Matsumoto and T. Baba, J. Lightwave Technol. 22, 917 (2004).

[20] T. Baba and D. Ohsaki, Jpn. J. Appl. Phys., Part 1 40, 5920 (2001).

[21] J. Witzens, M. Hochberg, T. Baehr-Jones, and A. Scherer, Phys. Rev. E 69, 046609 (2004).

[22] R. A. Shelby, D. R. Smith, S. C. Nemat-Nasser, and S. Schultz, Appl. Phys. Lett. 78, 489 (2001).

[23] C. G. Parazzoli, R. B. Greegor, J. A. Nielson, M. A. Thompson, K. Li, A. M. Vetter, M. H. Tanielian, and D. C. Vier, Appl. Phys. Lett. 84, 3232 (2004).

[24] S. Foteinopoulou, E. N. Economou, and C. M. Soukoulis, Phys. Rev. Lett. 90, 107402 (2003).

[25] E. Cubukcu, K. Aydin, E. Ozbay, S. Foteinopoulou, and C. M. Soukoulis, Nature (London) 423, 604 (2003).

[26] J. B. Pendry, Contemp. Phys. 45, 191 (2004).

[27] A. Taflove, Computational Electrodynamics-The FiniteDifference Time-Domain Method (Artech House, Norwood, MA, 1995). 